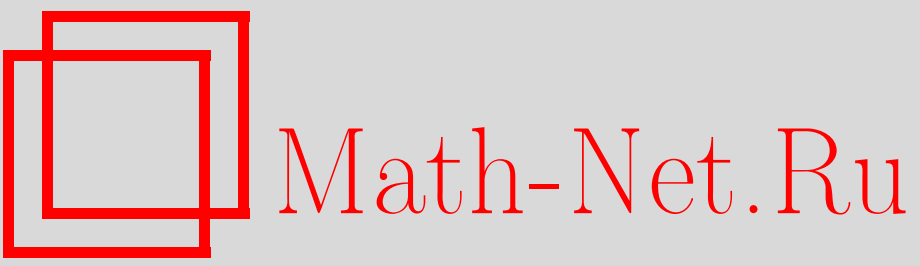

Л. В. Сабинин, О диассоциативности гладких моноальтернативных луп, УМН, 1996, том 51, выпуск 4, 169-170

DOI: https://doi.org/10.4213/rm1008

Использование Общероссийского математического портала Math-Net.Ru подразумевает, что вы прочитали и согласны с пользовательским соглашением

http://www.mathnet.ru/rus/agreement

Параметры загрузки:

IP : 18.234 .197 .8

26 апреля 2023 г., 12:37:04 


\title{
О ДИАССОЦИАТИВНОСТИ ГЛАДКИХ МОНОАЛЬТЕРНАТИВНЫХ ЛУП
}

\author{
Л.В. САБИнин
}

В заметке показано, что любая локальная $C^{2}$-гладкая моноальтернативная (в частности, аналитическая альтернативная) лупа диассоциативна.

1. Лупа $\langle Q, \cdot, \varepsilon\rangle$, наделенная унарными операциями $x \mapsto t x(x \in Q, t \in \mathbb{R})$, называется моноальтернативной, если $t x \cdot(u x \cdot y)=(t+u) x \cdot y,(y \cdot u x) \cdot t x=y \cdot(u+t) x$, для любых $x, y \in Q$; $t, u \in \mathbb{R}$.

2. ЗАмЕчАНИЕ. Для аналитической лупы свойство альтернативности

$$
x \cdot(x \cdot y)=(x \cdot x) \cdot y, \quad(y \cdot x) \cdot x=y \cdot(x \cdot x)
$$

влечет моноальтернативность [1].

3. Далее мы рассматриваем $C^{2}$-гладкие моноальтернативные лупы. В локальных координатах такие лупы описываются уравнениями [2]

$$
\begin{array}{ll}
\frac{d(x \cdot t y)^{i}}{d t}=A_{j}^{i}(x \cdot t y)\left(\operatorname{Exp}^{-1} y\right)^{j}, & (x \cdot 0 y)^{i}=x^{i} \\
\frac{d(u x \cdot y)^{i}}{d t}=\alpha_{j}^{i}(u x \cdot y)\left(\operatorname{Exp}^{-1} x\right)^{j}, & (0 x \cdot y)^{i}=y^{i},
\end{array}
$$

где

$$
A_{j}^{i}(x)=\left[\frac{\partial(x \cdot y)^{i}}{\partial y^{j}}\right]_{y=\varepsilon}, \quad \alpha_{j}^{i}(y)=\left[\frac{\partial(x \cdot y)^{i}}{\partial x^{j}}\right]_{x=\varepsilon}, \quad i, j=1, \ldots, \operatorname{dim} Q,
$$

a $\operatorname{Exp}: \zeta \in T_{\varepsilon} Q \mapsto \operatorname{Exp} \zeta \in Q$ определяется диффференциальными уравнениями

$$
\frac{d(\operatorname{Exp} t \zeta)^{i}}{d t}=A_{j}^{i}(\operatorname{Exp} t \zeta) \zeta^{j}, \quad \operatorname{Exp}(0)=\varepsilon,
$$

или, эквивалентно, в силу моноалштернативности, уравнениями

$$
\frac{d(\operatorname{Exp} t \zeta)^{i}}{d t}=\alpha_{j}^{i}(\operatorname{Exp} t \zeta) \zeta^{j}, \quad \operatorname{Exp}(0)=\varepsilon
$$

4. Скобки Ли линейно независимых векторных полей $A_{i}(x)=A_{i}^{j}(x) \frac{\partial}{\partial x^{j}}$ и $\alpha_{i}(y)=\alpha_{i}^{j}(y) \frac{\partial}{\partial y^{j}}$ однозначно определяют структурные функции $c_{q s}^{p}$ и $\bar{c}_{q s}^{p}$

$$
\left[A_{i}, A_{j}\right](x)=c_{i j}^{k}(x) A_{k}(x), \quad\left[\alpha_{i}, \alpha_{j}\right](y)=\bar{c}_{i j}^{k}(y) \alpha_{k}(y) .
$$

5. Следуя [2, гл. 7], можно ввести левую касательную алгебру $\langle V, *\rangle, V=T_{\varepsilon} Q$, лупы $\langle Q, \cdot, \varepsilon\rangle$ с бинарным умножением

$$
\xi * \eta=\xi^{i} c_{i j}^{k}(\operatorname{Exp} \xi) \eta^{j}\left(\partial_{k}\right)_{\varepsilon} .
$$

Аналогично определяется правая касательная алгебра $\langle V, \nabla\rangle$.

Согласно [2, гл. 7], в моноальтернативном случае

$$
\xi^{i} c_{i j}^{k}(\operatorname{Exp} \xi)=\xi^{i} c_{i j}^{k}, \quad c_{i j}^{k}=c_{i j}^{k}(\varepsilon)
$$

Аналогично,

$$
\xi^{i} \bar{c}_{i j}^{k}(\operatorname{Exp} \xi)=\xi^{i} \bar{c}_{i j}^{k}, \quad \bar{c}_{i j}^{k}=\bar{c}_{i j}^{k}(\varepsilon)=-c_{i j}^{k} .
$$

Следовательно, можно использовать только операцию $*$, так как $\xi * \eta=\eta \nabla \xi$. 
6. Вводя $B_{j}^{i}(x)$ и $\beta_{j}^{i}(x)$ как обратные матрицы к $A_{j}^{i}(x)$ и $\alpha_{j}^{i}(x)$ соответственно, можно написать (см. [2, гл. 7]) дифференциальные уравнения, определяющие в нормальных координатах матрицы $B_{j}^{i}(x)$ и $\beta_{j}^{i}(x)$ при помощи $c_{j k}^{i}$. В матричной форме

$$
\begin{aligned}
\frac{d t \overline{\bar{B}}(t a)}{d t}=\overline{\overline{\mathrm{Id}}}-(\overline{\overline{\operatorname{ad} a}})[t \overline{\bar{B}}(t a)], \quad[t \overline{\bar{B}}(t a)]_{t=0}=0, \\
\frac{d t \overline{\bar{\beta}}(t b)}{d t}=\overline{\overline{\mathrm{Id}}}+(\overline{\overline{\operatorname{ad} b}})[t \overline{\bar{\beta}}(t b)], \quad[t \overline{\bar{\beta}}(t b)]_{t=0}=0
\end{aligned}
$$

где $b^{i}=\operatorname{Exp}^{i}(b), \operatorname{ad} b: x \mapsto b * x$.

Поэтому

$$
\overline{\bar{B}}(a)=\frac{e^{-\overline{\overline{\mathrm{ad} a}}}-\overline{\overline{\mathrm{Id}}}}{(-\overline{\overline{\mathrm{ad} a}})}, \quad \overline{\bar{\beta}}(b)=\frac{e^{-\overline{\overline{\mathrm{ad} b}}}-\overline{\overline{\mathrm{Id}}}}{\overline{\overline{\mathrm{ad} b}}} .
$$

Отсюда, в частности, следует аналитичность $\overline{\bar{B}}(a)$ и $\overline{\bar{\beta}}(b)$, что вместе с (1) влечет аналитичность операции $(x, y) \mapsto x \cdot y$ лупы $\langle Q, \cdot, \varepsilon\rangle$ в нормальных координатах.

7. Рассмотрим $K^{i}(a, b)=\left[\frac{\partial^{4}(u a \cdot t b)^{i}}{(\partial u)^{2}(\partial t)^{2}}\right]_{t=u=0}$ в нормальных координатах. В силу моноальивности,

$$
u a \cdot t b=\left[(t b)^{-1} \cdot(u a)^{-1}\right]^{-1}=-[(-t b) \cdot(-u a)],
$$

Из (11) следует, что

$$
K^{i}(a, b)+K^{i}(b, a)=0 .
$$

Подсчитав $K^{i}(a, b)$ в нормалњных координатах, получаем

(13) $K(a, b)=\left(2 \beta_{1} \beta_{2}-2 \beta_{3}\right)\{b *[a *(b * a)]\}+\left(2 \beta_{3}-\beta_{1} \beta_{2}\right)\{a *[b *(a * b)]\}, \quad \beta_{1} \beta_{2} \beta_{3} \neq 0$, где $\beta_{1}, \beta_{2}, \beta_{3}$ - числа Бернулли.

Далее, (12) и (13) дают

$$
J(a, b, a * b)=0, \quad a, b \in V=T_{\varepsilon} Q
$$

$(J(\xi, \eta, \zeta) \stackrel{\text { def }}{=} \xi *(\eta * \zeta)+\zeta *(\xi * \eta)+\eta *(\zeta * \xi))$.

Мы получили следующее предложение.

ПредЛОжение 1. Пусть $\langle Q, \cdot, \varepsilon\rangle-C^{2}$-гладкая локальная моноальтернативная лупа. Тогда ее касательная алгебра $\langle V, *\rangle, V=T_{\varepsilon} Q$, моноальтернативна, т.е. $J(\xi, \eta, \xi * \eta)=0$.

8. Согласно [6] кососимметричная алгебра моноальтернативна, если и только если она бинарно-лиева. т.е. если любые два ее элемента порождают подалгебру Ли, см. [5].

Известно также, [5], что $C^{2}$-гладкая лупа диассоциативна (т.е. любые два ее элемента порождают подгруппу Ли), если и только если ее касательная алгебра бинарно-лиева.

В силу сказанного получаем

ПРЕДЛОЖЕНИЕ 2. Любая $C^{2}$-гладжая моноальтернативная лупа диассоциативна.

9. ЗАмЕчАниЕ. В работе [7] показано, что локальная аналитическая альтернативная лупа со свойством эластичности $x \cdot(y \cdot x)=(x \cdot y) \cdot x$ диассоциативна. Предложение 2 вместе с [1] показывает, что условие эластичности в [7] излишне.

\section{СПИСОК ЛИТЕРАТУРЫ}

[1] Сабинин Л. В., Михеев П.О. // Проблемы теории тканей и квазигрупп. Калинин: Изд-во Калининского ун-та, 1985. С. 72-75. [2] Сабинин Л. В. Аналитические квазигруппы и геометрия. М.: Университет Дружбы народов, 1991. [3] Белоусов В. Д. Основы теории квазигрупп и луп. М.: Наука, 1967. [4] Белоусов В. Д. Элементы теории квазигрупп и луп. Кишинев: Изд-во Кишиневского ун-та, 1981. [5] Мальцев А. И. // Матем. сб. 1955. Т. 36(78). № 3. С. 569-576. [6] Гайнов А. Т. // УМН. 1957. Т. 12. С. 141-146. [7] Михеев П. О. // Матем. заметки. 1993. Т. 54. № 5. С. $72-77$. 